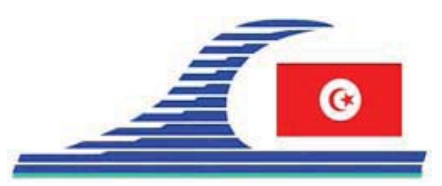

Conférence Méditerranéenne Côtière et Maritime

EDITION 1, HAMMAMET, TUNISIE (2009)

Coastal and Maritime Mediterranean Conference

Disponible en ligne - http://www.paralia.fr-Available online

\title{
Evolution des pratiques de suivi topo-bathymetrique du littoral en Languedoc-Roussillon : l'utilisation du lidar
}

\author{
Cyril VANROYE $^{1}$
}

1. Direction Régionale de l'Equipement du Languedoc-Roussillon

Service des Espaces Littoraux, Unité Aménagements et Risques Littoraux 520, allée Henri II de Montmorency, 34064 Montpellier Cedex 2, France

cyril.vanroye@developpement-durable.gouv.fr

\section{Résumé :}

La connaissance de la morphologie du littoral et de son évolution est à la base de la gestion des risques littoraux que sont l'érosion et la submersion marine. Le littoral du Languedoc-Roussillon soumis à une importante dynamique sédimentaire fait l'objet d'actions de suivis réguliers de la part des gestionnaires, en premier lieu l'Etat au travers de ces services déconcentrés. Après avoir utilisé des techniques de suivi topobathymétriques «classiques » qui ont donné les premiers éléments de connaissance mais présentent des limites, la Direction Régionale de l'Equipement du LanguedocRoussillon a désormais recours au LIDAR bathymétrique à l'échelle régionale. Cette évolution vient de prendre un tournant décisif au cours de l'année 2009 avec la réalisation de la première campagne d'acquisition de données dans le Golfe du Lion couvrant une superficie de plus de $470 \mathrm{~km}^{2}$ et qui a mobilisé un investissement de 765 $000 €$. Les résultats obtenus sont sans précédent en terme de qualité et de densité de couverture et vont contribuer à l'amélioration des connaissances des phénomènes à l'oeuvre sur la côte sableuse languedocienne. Cette opération est emblématique du positionnement de l'Etat français dans les actions liées à l'adaptation au changement climatique et à la mise en oeuvre du Grenelle de la Mer.

\section{Mots-clés :}

LIDAR bathymétrique - Hydrographie - Risques côtiers

\section{Le suivi du littoral en Languedoc-Roussillon}

La façade littorale du Languedoc-Roussillon, d'un linéaire d'environ $220 \mathrm{~km}$, concerne 30 communes sur quatre départements.

Elle présente deux caractéristiques majeures et uniques sur le territoire métropolitain : la côte est très majoritairement sableuse avec près de $190 \mathrm{~km}$ de plages et est constituée de terrains de très faibles altimétrie, ponctués de complexes lagunaires salés très étendus. L'occupation humaine y est récente et s'est développée dans le cadre d'une vaste opération d'aménagement dans les années 60. La dynamique sédimentaire y est très forte et les phénomènes d'érosion très actifs. Près de 30\% du linéaire de côte est DOI: $10.5150 / \mathrm{cmcm} .2009 .076-3$ 
considéré en érosion. Aussi, le suivi de l'évolution du littoral a très tôt constitué un sujet de préoccupation pour les gestionnaires de littoral. Pendant près de vingt ans, le Service Maritime et de Navigation du Languedoc-Roussillon (S.M.N.L.R.) a réalisé un suivi régulier du littoral. Ces levés ont en outre été associés à des levés bathymétriques réalisés sur certains secteurs en fonction des besoins ainsi que le suivi du trait de côte au moyen de G.P.S. différentiel. Ce suivi consistait en la réalisation de profils topographiques et bathymétriques le long du littoral. Près de 200 profils ont ainsi été réalisés. Ils ont fait l'objet au début des années 2000 d'une numérisation qui assure aujourd'hui la pérennité de ces données et constitue une source d'information unique sur l'évolution du littoral. Ces profils ont été utilisés dans la plupart des études générales de protection réalisées et dans les études scientifiques menées ces dernières années. Cependant, l'évaluation de cette méthodologie de suivi a mis en évidence certains écueils notamment : des décalages temporels importants entre interventions topographiques et bathymétriques (ou l'absence totale de l'un ou de l'autre dans certains cas), des variations dans la localisation des profils, l'aspect partiel et très ponctuel de ces suivi. Aussi, compte tenu des enjeux régionaux associés à l'évolution du littoral, il s'est avéré nécessaire de rechercher une solution technique permettant de remédier à ces inconvénients.

\section{L'expérience acquise dans le cadre du projet européen BEACHMED-e}

Les attentes en terme de suivi morphologique du littoral sont : visualiser avec précision la morphologie du littoral, couvrir des surfaces importantes sans décalage temporel, assurer la continuité dans la zone des petits fonds (entre $1 \mathrm{~m}$ et 1,50 m). L'objectif final est de mettre en évidence la dynamique sableuse et de quantifier les déplacements sédimentaires. Les évolutions technologiques et l'apparition de systèmes opérationnels de LIDAR (Light Detection And Ranging) bathymétriques dans les années 1990 ont permis d'orienter la réflexion vers l'utilisation de cette technologie. Le LIDAR est une technique de télédétection basée sur l'émission-réception d'un faisceau laser. Dans le cas de l'utilisation pour l'hydrographie, on a recours à l'émission de deux faisceaux : un rouge (longueur d'onde : $1064 \mathrm{~nm}$ ) et un vert (longueur d'onde : $532 \mathrm{~nm}$ ). Le faisceau rouge est réfléchi par la surface de l'eau et le faisceau vert est pour partie réfléchi par la surface de l'eau mais aussi par le fond. Les signaux réfléchis sont captés à bord de l'avion en vol. La mesure de la hauteur d'eau est déduite du temps séparant les échos de la surface et du fond (BALOUIN \& HEURTEFEUX, 2007).

L'acquisition de données est réalisée à bord de l'avion qui réalise des lignes de vol suivant des directions prédéterminées. Le capteur réalise un balayage afin de couvrir la zone de part et d'autre de la ligne de vol. Dans le cadre de l'opération cadre BEACHMED-e (2009), (programme INTERREG III c), la Direction Régionale de l'Equipement du Languedoc-Rousillon (DRE LR), le Conseil Général de l'Hérault et 
l'Entente Départementale de Démoustication Méditerranée (EID) ont procédé à un vol LIDAR dans le but d'étudier la faisabilité de l'utilisation de cette technique.

Cette opération a donné lieu à un levé dans le Golfe d'Aigues-Mortes (plages de Frontignan, Palavas et Carnon) en avril 2007, d'une superficie totale de $47 \mathrm{~km}^{2}$. Le capteur utlisé est le laser mixte Hawke Eye II (BLOM, site Web). Sur ce levé, les comparaisons effectuées avec des données acquises avec des techniques classiques (échosondeur) ont établi des écarts sur la mesure verticale de l'ordre de $15 \mathrm{~cm}$ (BEACHMED-e, 2009). Les résultats de ce levé ont conduit la DRE LanguedocRoussillon à envisager une nouvelle orientation pour ces interventions de suivi du littoral et à décider de recourir au LIDAR bathymétrique.

\section{Un premier levé régional en 2009}

Le premier levé régional par LIDAR bathymétrique a ainsi été réalisé sous maîtrise d'ouvrage de la Direction Régionale de l'Equipement LR. L'acquisition des données s'est déroulée du 24 août 2009 au 8 septembre 2009 et la validation des données est attendue pour fin 2009. Le prestataire en charge de la réalisation du levé et du traitement des données est le groupement d'entreprise EUROSENSE - FUGRO LADS. Le levé couvre la quasi-totalité du littoral régional et a suivi le découpage des cellules sédimentaires. La superficie levée est de $303 \mathrm{~km}^{2}$.

Le système d'acquisition est le LADS Mk II (FUGRO LADS, Site Web) et la résolution choisie est $5 \mathrm{~m} * 5 \mathrm{~m}$. La profondeur minimale à atteindre prescrite par le cahier des charges était l'isobathe $10 \mathrm{~m}$ mais la profondeur $20 \mathrm{~m}$ a été atteinte de manière générale au cours du levé. A terre la couverture s'étend jusqu'au cordon dunaire.

En outre, la DRE LR s'est rapprochée du Service Hydrographique et Océanographique de la Marine (SHOM) référent dans le domaine de la bathymétrie afin d'assurer le rôle d'expert et de contrôler les données finales. Les données seront intégrées dans le projet de Référentiel Grande Echelle continu terre-mer porté par l'IGN et le SHOM, LITTO3D ${ }^{\circledR}($ IGN, site web ; SHOM, site web). Le contrôle et la réception des données sera assurée par le SHOM au moyen de zones de contrôles volées systématiquement au début et à la fin de chaque acquisition. Une comparaison sera aussi effectuée avec des levés topographiques et bathymétriques réalisées concomittament sur les plages du Golfe d'Aigues-Mortes. Le financement a été intégralement pris en charge par l'Etat à hauteur de $765000 €$. Le coût de cette campagne se situe aux alentours de $2500 € / \mathrm{km}^{2}$. Ces coûs sont à comparer avec les prix récemment obtenus dans le cadre d'un appel d'offres pour l'acquisition de données au moyen de techniques classiques : pour une surface à couvrir de $20 \mathrm{~km}^{2}$, la moyenne des 9 prix les plus bas se situe à $2200 € / \mathrm{km}^{2}$ et dans une fourchette de prix de $1500 € / \mathrm{km}^{2}$ à $2600 € / \mathrm{km}^{2}$. Il convient aussi de mettre en regard les vitesses d'acquisition, l'acquisition des données sur les $303 \mathrm{~km}^{2}$ auront nécessité 56 heures de vol. 


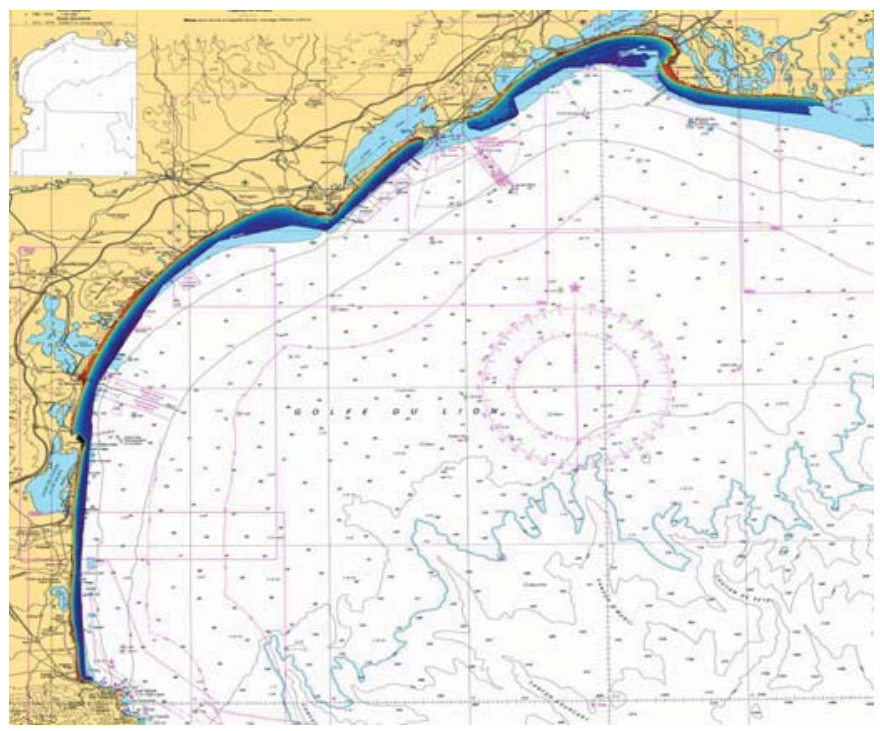

Figure 1. Levé LIDAR 2009 dans le Golfe du Lion.

\section{Références}

BALOUIN Y., HEURTEFEUX H. (2007). Utilisation de la technologie du Lidar bathymétrique pour le suivi du littoral: retours d'expériences sur le littoral Méditerranéen. Revue française de photogrammétrie et de télédétection, 2007.

BEACHMED-e (2009). Rapports relatifs au sous-projet OPTIMAL. [URL http://www.beachmed.it/ ]

FUGRO LADS (site web). http://www.fugrolads.com/ladsmkII.htm

IGN ((site web).

http://www.ign.fr/institut/documentArticle.do?idDoc $=5591865 \&$ indexRoot=8\&indexChild $=5$ SHOM (site web). http://www.shom.fr/ 